Portland State University

PDXScholar

Computer Science Faculty Publications and

Presentations

Computer Science

$10-1998$

Theories of Structure versus Theories of Change

Melanie Mitchell

Santa Fe Institute

Follow this and additional works at: https://pdxscholar.library.pdx.edu/compsci_fac

Part of the Computational Neuroscience Commons, and the Ecology and Evolutionary Biology

Commons

Let us know how access to this document benefits you.

Citation Details

Mitchell, Melanie. "Theories of structure versus theories of change." Behavioral and Brain Sciences 21.05 (1998): 645-646.

This Article is brought to you for free and open access. It has been accepted for inclusion in Computer Science Faculty Publications and Presentations by an authorized administrator of PDXScholar. Please contact us if we can make this document more accessible: pdxscholar@pdx.edu. 
gent actions by exploiting the physical characteristics of its own body and its environment. Cognition is redrawn as a notion centered around intelligent behavior - or adaptive organismenvironment interactions. The newfound importance of timing problems in cognition is a direct corollary of this organismenvironment interaction perspective. For successful behavior, it is not only important to decide what should be done, but also in what time-window certain initiated actions will be successful. What cognition consists of is increasingly answered in terms of a capacity for intelligent behavior rather than abstract thought. All of this is mentioned by van Gelder as supporting the DH, but these developments would still stand if someone would prove that they were perfectly reconcilable with the $\mathrm{CH}$.

The derived importance of the DH becomes particularly obvious when a specific theoretical issue is considered: the use of representations in cognitive science. The classical story is that the outward behavior of cognitive agents results from an internally represented program that initiates and guides the external behavioral events, or the output for short - of course always in conjunction with auxiliary feedback loops and other peripheral mechanisms. Van Gelder classifies this general idea with the $\mathrm{CH}$, whereas the DH would provide different options for the use of representations or even their total abandonment.

We find the current dominant interpretation of representation highly problematical; radical changes are needed to fit this notion in cognitive science. Dynamical modeling, however, fails to provide the necessary leverage to initiate a change in the use of representations in cognitive theorizing (Keijzer \& Bem 1996). The DH can be perfectly reconciled with a classical interpretation of representations and the $\mathrm{CH}$ can be combined with different kinds of representation. Any change in the notion of representation will derive from changes in our ideas about how representational entities function in cognitive theories (Keijzer, in press). The DH is too general to take the leading role here.

The really important issue for cognitive science right now is to extend our understanding of cognitive systems. Whether we will prefer to call these systems computational or dynamical will be a pragmatic decision, depending on what seems to be the most natural and efficient way to explicate and formalize these ideas. Given the direction of current insights into cognitive processes, it seems possible and even plausible that this will ultimately lead to something like the DH. First, however, it should be made clear what all these dynamics are supposed to be the dynamics of.

\section{Theories of structure versus theories of change}

\section{Melanie Mitchell \\ Santa Fe Institute, Santa Fe, NM 87501; mm@santafe.edu \\ www.santafe.edu/ mm}

\begin{abstract}
The dynamics/computation debate recalls a similar debate in the evolutionary biology community concerning the relative primacy of theories of structure versus theories of change. A full account of cognition will require a rapprochement between such theories and will include both computational and dynamical notions. The key to making computation relevant to cognition is not making it analog, but rather understanding how functional information-processing structures can emerge in complex dynamical systems.
\end{abstract}

Is cognition about change or is it about structure? Van Gelder clearly thinks that change is the essence: he champions dynamical systems theory because "dynamicists are interested, in the first instance, in how things change; states are the medium of change, and have little intrinsic interest. Computationalists, by contrast, focus primarily on states; change is just what takes you from one state to another. ... Computationalists focus on internal structure." (sects. 4.2.3.1 and 4.2.3.2)
Van Gelder's formulation of this opposition - between dynamics as focused on state change and computation as focused on state internal structure - brings to mind a similar debate that has gone on for years in the evolutionary biology community, and whose resolution will, I believe, be instructive for the dynamics/computation debate in cognitive science. What accounts for the biological phenomena we observe in the world? The predominant explanatory framework has been neo-Darwinism, a theory of change par excellence (inherited random change from one generation to the next leads to adaptation by natural selection). However, some evolutionary theorists have questioned the adequacy of classical neo-Darwinism as either an explanatory or a predictive theory, and argue instead for the primacy of historical contingency (Gould 1989a) or the self-organization of biological structure not due to natural selection (Fontana \& Buss 1994; Goodwin 1990; Kauffman 1993). These "historicists" and "structuralists" are the connectionists of the evolutionary biology community - the people questioning the classical orthodoxy.

The selectionist/historicist/structuralist debate was summarized by Seilacher in his triangle of causal determinants of form (Seilacher 1991), and has been discussed at length by Gould (1989b), among others. It is becoming increasingly clear, however, that the stark oppositions posited among these three frameworks are not only false ones, but they are hindering progress in evolutionary theory. The purely structuralist theories do not explain how structures can be significantly changed in evolution, and the purely selectionist theories do not explain what intrinsic driving forces and constraints there are on the formation of biological structures. What is needed is a theory that incorporates both change and structure. ${ }^{1}$

Similarly, in cognitive science we have theories of change and movement ("dynamical" approaches): how robots walk in a stable manner (Beer 1995b), how babies reach and grasp (Thelen \& Smith 1994), how people move from a condition of uncertainty to making a decision (Busemeyer \& Townsend 1993). These theories, however, do not explain the information-processing content of the states over which change is occurring; they either address tasks that do not require complex information processing or they assume high-level information-related primitives a priori. For example, in Busemeyer and Townsend's Decision Field Theory, described in the target article, information-loaded notions such as "positive and negative consequences," "attention and shift of attention," "preferences," and "motivation" are atomic primitives (Busemeyer \& Townsend 1993), and the theory does not attempt to explain how these are implemented or why a particular decision-maker might have one version of them instead of another.

Likewise, in cognitive science we have theories of structure ("computational" approaches) that make statements about the information-processing structure of concepts, representations, and beliefs (e.g., semantic networks, neural networks, schemata, Bayesean belief networks, fuzzy logic, theorem provers). As van Gelder points out, most of these theories assume that information processing consists of the manipulation of explicit, static symbols rather than the autonomous interaction of emergent, active ones (Hofstadter 1985). Such theories typically cannot explain what driving forces and constraints there are on how the system in question can change, what trajectories it can take, and how the highlevel symbols can emerge from a lower-level substrate.

Thus, as in evolutionary biology, cognitive science needs rapprochements between theories of change and theories of structure. Attempts at such rapprochements are coming from many sectors, in particular from research on "complexity," in which dynamics, computation, and adaptation are beginning to be viewed in a more unified framework. For example, in our work on emergent computation in cellular automata, my colleagues and I have shown how active representations and functional information processing can emerge from interactions among dynamical systems, an environment, and an adaptive evolutionary process (Crutchfield \& Mitchell 1995; Das et al. 1994; 1995). This work is a pre- 
liminary step in understanding how useful computation can be embedded in a complex dynamical system; it is one attempt at (as van Gelder puts it) "dramatically reconceiving how [complex internal structures] might be instantiated" in such a system (sect. 6.9). In the end, van Gelder seems to agree that computational notions - albeit of a nontraditional kind - might be important for cognitive science: "the DH can embrace the idea that cognitive processes are computational” (sect. 6.3). However, unlike van Gelder, I do not believe that it is the digital/analog distinction that is key for making computation relevant for cognition; instead, I think progress will come from understanding how functional information-processing structures can emerge in spatially extended dynamical systems with no central control, no globally accessible memory, and limited communication among components. Computer science is gradually moving in this direction, and I believe that many useful synergies between computation theory and cognitive science will arise in the near future.

Van Gelder's answer to objection 6.7 ("not as cognitive") is that according to the DH "cognitive agents are dynamical systems of quite special kinds" (sect. 6.7, para. 2). I will venture to say that they are dynamical systems in which the states and state trajectories can and must be interpreted in functional, informational, and information-processing terms, and the computational notions will be necessary as well as dynamical notions for constructing a full account.

\section{NOTE}

1. This formulation of the evolution debates was given to me by evolutionist Daniel McShea (personal communication). McShea's formulation was elaborated by Crutchfield (1994), who proposes a particular computation-theoretic notion of structure ("computational mechanics of nonlinear processes") and a related mechanism for the transformation of structure ("hierarchical machine reconstruction"). Crutchfield suggests that a unified theory of these two processes might be termed "evolutionary mechanics," which he proposes as a general theory of "emergence."

\section{Nature's subtlety undermines the empirical relevance of both dynamical and computational hypotheses}

\author{
Gregory R. Mulhauser \\ Cognitive Systems Research, Applied Research and Technology, British \\ Telecom Laboratories, Martlesham Heath IP5 3RE, England \\ scarab@info.bt.co.uk www.labs.bt.com/people/mulhaug
}

\begin{abstract}
Technical hitches mar van Gelder's proposed map of the conceptual landscape, particularly with respect to descriptive levels and the trio of instantiation, realisation, and implementation. However, for all the formal quibbles, van Gelder is onto something important; the tension he notes between computationalism and a dynamical alternative threatens to transform the way we conduct cognitive science research.
\end{abstract}

The relationships of instantiation, realisation, and implementation sit at the heart of van Gelder's explication of systems in general and of the computational and dynamical hypotheses in particular. As presented, however, these relationships do not support the tidy dynamical versus computational carving up of the world that van Gelder's project requires.

A central difficulty is that any finite sequence of empirical measurements can in principle be described mathematically in infinitely many distinct ways (the curve fitting of sect. 6.6). Any given object instantiates not only a great many systems (sect. 3.1), but infinitely many. Likewise for implementation: the weak constraint that higher level variables are somehow constructed (sect. 3.1) from those at lower levels leaves the relationship easy prey to "gerrymandering," or wild ad hoc perversions of the spirit of van Gelder's idea that satisfy the letter of his account in unexpected ways. Such inadequately constrained criteria render van Gelder's critically important claims about causal organisation awkward to evaluate. They also invite inconveniences such as Putnam's (1988, pp. 120-25) proof that every ordinary open physical system trivially implements ("instantiates," for van Gelder) every abstract finite state automaton.

Perhaps van Gelder believes that the fundamental relationships can be buttressed easily to support the required distinctions and, crucially, to deliver the big prize of causal organisation that grounds the project. Optimism here places van Gelder in good contemporary company: Chalmers, too, believes that his own single-paragraph description of implementation (Chalmers 1996, p. 318) captures causal organisation. However, neither author acknowledges that the subtleties of pinning down causal organisation occupy entire books (Kitcher \& Salmon 1989; Nagel 1961; Rescher 1970; Skryms 1980), having puzzled philosophers of science at least since the inception of the "covering law" model in Hempel and Oppenheim's (1948) seminal paper. The nuances of the problem trace their heritage back, ironically, to David Hume's own scepticism about "necessary connexions" in section VII of his 1748 Enquiry Concerning Human Understanding.

Although appeals to the compactness of a description do offer useful constraints, sufficient to preclude some gerrymandering, the concept is tricky. Van Gelder's account of dynamical systems (sect. 3.3, items 1 and 3), hides another glitch: in the general case, the question of how succinctly something may be expressed is formally undecidable. The subject merits a whole field of its own, called algorithmic information theory (Chaitin 1987). At least one usefully nontrivial account of implementation can be constructed with the help of a somewhat tedious information theoretic analysis (Mulhauser 1998), but the resulting view lends no support to a strict demarcation between dynamics and computation.

These quibbles arise more from general problems in the philosophy of science than from the dynamical hypothesis; in fact, the account of computation van Gelder draws from Haugeland (1985) stands, if anything, on even shakier metaphysical footing. Pathological weaknesses in his appeal to correspondence frequently serve for target practice by philosophers such as Sterelny (1990). The upshot? Neither the dynamical nor the computational hypotheses is empirically meaningful in any strong sense: the underlying concepts are too impoverished to support the necessary taxonomy. (Similarly, "all oceans contain fish" would not be an empirical hypothesis if our concept of "fish" were trivially open to philosophical predation.) More relevant to the direction of empirical work is not how different models are classified, but how they enrich our understanding.

Indeed, the rhetorical elegance of van Gelder's treatment should not obscure the fact that both dynamical and computational descriptions, perhaps at different levels, may be invaluable for understanding the same physical object. Real cognitive models rarely fall into a clean explanatory cascade anyway, neatly subsuming one another in relationships of logical supervenience; more often, they are complementary, "horizontally" related, and largely incommensurable. Therefore, van Gelder's persistence in referring to the highest relevant level of causal organisation seems as odd as referring to the highest relevant integer for doing number theory; any particular level or integer may be too high, too low, or just right, depending on the questions being asked. A privileged set of the "right" questions no more exists in cognitive theory than in number theory.

By way of analogy, consider possible explanations of my Macintosh's behaviour while it displays pictures of balls bouncing across the screen. We could start at the (thoroughly dynamical) quantum level, appealing to properties of semiconductor junctions to explain how changes in the computer's internal components and ultimately its display screen occur so reliably and consistently, or we might pick a software level to explain computationally how digital values are copied to a display buffer and make their way to the screen. Alternatively, the best predictor of displayed ball trajectories is probably some class of dynamical systems describing elastic collisions. And in trying to understand "digital" Macintosh balls 